\title{
Exact solutions of Dirichlet type problem to elliptic equation, which type degenerates at the axis of cylinder. I
}

\section{Stasys Rutkauskas*}

\section{"Correspondence:}

stasys.rutkauskas@mii.vu.lt Institute of Mathematics and Informatics of Vilnius University, Akademijos str. 4, Vilnius, 08863 , Lithuania

\section{Springer}

\begin{abstract}
In this article, an elliptic equation, which type degenerates (either weakly or strongly) at the axis of a 3-dimensional cylinder, is considered. The statement of a Dirichlet type problem in the class of smooth functions is given and, subject to the type of degeneracy, the exact classical solutions are obtained. The uniqueness of the solutions is proved and the continuity of the solutions on the line of degeneracy is discussed.
\end{abstract}

Keywords: degenerate elliptic equations; boundary value problems; Dirichlet type problem

\section{Introduction and statement of the problem}

We consider the equation

$$
u_{z z}+r^{2 \alpha} \Delta u-c u=0, \quad \alpha>0,
$$

in the cylinder $Q=\left\{x^{2}+y^{2}<R^{2}, 0<z<H\right\}$. Here $r=\sqrt{x^{2}+y^{2}}, \Delta=\frac{\partial^{2}}{\partial x^{2}}+\frac{\partial^{2}}{\partial y^{2}}$ is Laplace's operator, $c \geq 0$ is a real constant.

Evidently, equation (1) is elliptic outside of the line $r=0$ and its type degenerates at this line, i.e., at the axis of cylinder $Q$. Since the parameter $\alpha>0$ is undetermined, the degeneracy can be either regular $(\alpha \leq 1)$ or irregular $(\alpha>1)$. The Dirichlet type problems for the elliptic systems, which are irregularly degenerate at the inner point of a considered domain, are developed, e.g., in [1-3]. It is advisable to mention the work [4-6] related with the subject of this article, too.

In comparison with the degeneracy of elliptic equations at an inner point, the main difficulty in the consideration of the Dirichlet problem to equation (1) is related with the formulation of the boundary value conditions on the bases of cylinder $Q$, to be precise, with the behavior of boundary functions at the points $P_{0}(0,0,0)$ and $P_{H}(0,0, H)$ in which the line of degeneracy crosses the bases of cylinder $Q$. The Dirichlet problem to some particular cases of equation (1) are considered in [7] (the cases when $\alpha=1$ and 1/2) and in [8]. However, here is discussed only the case of boundary value conditions when the boundary functions are zero valued on the two bases of cylinder $Q$. In this paper, we consider the

(c) 2016 Rutkauskas. This article is distributed under the terms of the Creative Commons Attribution 4.0 International License (http://creativecommons.org/licenses/by/4.0/), which permits unrestricted use, distribution, and reproduction in any medium, provided you give appropriate credit to the original author(s) and the source, provide a link to the Creative Commons license, and indicate if changes were made. 
Dirichlet type problem to equation (1) with non-zero boundary value conditions on these bases.

It is convenient to introduce the cylindrical coordinates $r, \varphi, z(|\varphi| \leq \pi)$ in which equation (1) takes the shape

$$
u_{z z}+L(u)=0
$$

where

$$
L(u):=r^{2 \alpha}\left(\frac{\partial^{2} u}{\partial r^{2}}+\frac{1}{r} \frac{\partial u}{\partial r}+\frac{1}{r^{2}} \frac{\partial^{2} u}{\partial \varphi^{2}}\right)-c u .
$$

(Here we denote a solution $v(r, \varphi, z)=u(r \cos \varphi, r \sin \varphi, z)$ of equation (2) by $u(r, \varphi, z)$ again.)

Let us to introduce the following notations: $Q_{\delta}$ is a cylindric ring $Q \backslash\{0 \leq r \leq \delta<R, 0<$ $z<H\}, S=\{|\varphi| \leq \pi, 0<z<H\}, D$ is the disc $\{r<R,|\varphi| \leq \pi\}, D_{\delta}$ is the $\operatorname{ring} D \backslash\{0 \leq r \leq \delta<$ $R\}, K$ is the circle $\{r=R,|\varphi| \leq \pi\}, \bar{\Omega}$ is the closure of any domain $\Omega$. Routinely, we denote by $\mathbb{N}$ the set of natural numbers and by $\mathbb{N}_{0}$ the set of non-negative integer numbers, and by $C^{l}(\Omega)$ the class of functions of which the derivatives are continuous up to order $l$ in any domain $\Omega$.

Problem D1 Find the solution $u(r, \varphi, z)$ of equation (2) in the class of functions $C^{2}\left(Q_{0}\right) \cap$ $C\left(\bar{Q} \backslash\{r=0\}\right.$ ) (or, maybe, in the class $C^{2}\left(Q_{0}\right) \cap C(\bar{Q})$ ) which is bounded in $Q_{0}$ and satisfies the boundary value conditions

$$
\begin{aligned}
& u(R, \varphi, z)=0, \quad(\varphi, z) \in \bar{S}, \\
& u(r, \varphi,(i-1) H)=f_{i}(r, \varphi), \quad i=1,2,
\end{aligned}
$$

for $(r, \varphi) \in D_{0} \cup K$ (or, maybe, for $(r, \varphi) \in \bar{D}$ ), where $f_{i}$ are given continuous functions such that

$$
f_{i}(R, \varphi)=0 .
$$

(Besides, we assume that $f_{i}(r, \varphi), i=1,2$, are $2 \pi$-periodic in the $\varphi$ functions.)

The aim of the present paper is to discuss the well-posedness of the functions $f_{i}, i=1,2$, in the vicinity of the points $P_{0}$ and $P_{H}$, and to obtain the exact solutions of Problem D1 subject to the type of degeneracy of equation (2).

The Dirichlet problem

$$
u(R, \varphi, z)=f(\varphi, z), \quad u(r, \varphi,(i-1) H)=0, \quad i=1,2,
$$

to equation (2) is treated in the class of functions $C^{2}\left(Q_{0}\right) \cap C(\bar{Q})$ in [9]. Assuming that function $f$ is twice differentiable, here the representations of exact solutions of this problem are given in all cases of the degeneracy of equation (2).

\section{The spectrum properties of the operator $L$}

We consider the following eigenvalues problem. 
$\boldsymbol{E} \boldsymbol{V}$-problem Find the solutions $w(r, \varphi ; \lambda)$ of equation

$$
L(w)+\lambda w=0, \quad \lambda \in \mathbb{R},
$$

in the class of functions $C^{2}\left(D_{0}\right) \cap C(\bar{D} \backslash\{r=0\})$ (or, maybe, in the class $C^{2}\left(D_{0}\right) \cap C(\bar{D})$ ) satisfying the conditions

$$
w(R, \varphi ; \lambda)=0, \quad|w|<\infty \quad \text { in } D_{0} .
$$

Using the method of separate variables, we obtain the following partial solutions of equation (7):

$$
P_{m}(r ; \lambda) \times \begin{cases}\cos m \varphi, & m \in \mathbb{N}_{0} \\ \sin m \varphi, & m \in \mathbb{N}\end{cases}
$$

where $P_{m}(r ; \lambda)$ is the solution of the Sturm-Liouville problem (in the following we call it the $S L$-problem),

$$
\begin{aligned}
& r^{2} P^{\prime \prime}+r P^{\prime}+\left[(\lambda-c) r^{2(1-\alpha)}-m^{2}\right] P=0, \\
& P(R)=0, \quad|P(r)|<\infty \quad \text { on }(0, R] .
\end{aligned}
$$

Assume that $\alpha<1$. If $\lambda>c$, then equation (9) has only one bounded solution,

$$
P_{m}(r ; \lambda)=J_{\frac{m}{1-\alpha}}\left(\frac{\sqrt{\lambda-c}}{1-\alpha} r^{1-\alpha}\right)
$$

with the accuracy of a constant multiplier, whereas all other linear independent solutions are unbounded at the point $r=0$. (Here $J_{v}$ is the Bessel function of the first kind [10].) Let $\gamma_{m n}$ be the roots of the Bessel function $J_{\frac{m}{1-\alpha}}$, i.e. $J_{\frac{m}{1-\alpha}}\left(\gamma_{m n}\right)=0, n \in \mathbb{N}_{0}$. Choose the values of parameter $\lambda$ by the definition

$$
\lambda=\lambda_{m n}:=c+\gamma_{m n}^{2}(1-\alpha)^{2} R^{2(\alpha-1)} .
$$

Then the corresponding solutions

$$
P_{m n}(r):=P_{m}\left(r ; \lambda_{m n}\right)=J_{\frac{m}{1-\alpha}}\left(\gamma_{m n}\left(\frac{r}{R}\right)^{1-\alpha}\right), \quad n \in \mathbb{N}_{0}
$$

of equation (9) are such that $P_{m n}(R)=0$, obviously. Further, it follows from the properties of the Bessel functions $[10,11]$, that

$$
\begin{aligned}
& P_{0 n}(0)=1, \\
& P_{m n}(r)=\left(\frac{\gamma_{m n}}{2}\right)^{\frac{m}{1-\alpha}}\left(\frac{r}{R}\right)^{m}\left(1+O\left(r^{1-\alpha}\right)\right) \rightarrow 0 \quad \text { as } r \rightarrow 0, m \in \mathbb{N} .
\end{aligned}
$$


Hence, $P_{m n}(r)$ are continuous on the interval [0,R] eigenfunctions of $S L$-problem (9), (10) corresponding to their eigenvalues $\lambda_{m n}, n \in \mathbb{N}_{0}$, defined by (11). Then

$$
J_{\frac{m}{1-\alpha}}\left(\gamma_{m n}\left(\frac{r}{R}\right)^{1-\alpha}\right) \times \begin{cases}\cos m \varphi, & m \in \mathbb{N}_{0}, \\ \sin m \varphi, & m \in \mathbb{N},\end{cases}
$$

are the eigenfunctions of $E V$-problem (7), (8) corresponding to eigenvalues $\lambda_{m n}, n \in \mathbb{N}$.

In the case when $\alpha>1$, there exist two linearly independent solutions,

$$
P_{m}^{(1)}(r ; \lambda)=J_{\frac{m}{\alpha-1}}\left(\frac{\sqrt{\lambda-c}}{\alpha-1} r^{1-\alpha}\right), \quad P_{m}^{(2)}(r ; \lambda)=N_{\frac{m}{1-\alpha}}\left(\frac{\sqrt{\lambda-c}}{\alpha-1} r^{1-\alpha}\right)
$$

of equation (9), which are bounded at the point $r=0$ under condition $\lambda>c$. (Here $N_{v}$ is Bessel function of second kind or so called Neumann function [10].) Specifically, we have the following asymptotic expansions [11]:

$$
\begin{aligned}
& P_{m}^{(1)}(r ; \lambda)=p_{m \lambda} r^{\frac{\alpha-1}{2}} \cos \left(\frac{\sqrt{\lambda-c}}{\alpha-1} r^{1-\alpha}-\frac{\pi m}{2(\alpha-1)}-\frac{\pi}{4}\right)\left(1+O\left(r^{\alpha-1}\right)\right), \\
& P_{m}^{(2)}(r ; \lambda)=p_{m \lambda} r^{\frac{\alpha-1}{2}} \sin \left(\frac{\sqrt{\lambda-c}}{\alpha-1} r^{1-\alpha}-\frac{\pi m}{2(\alpha-1)}-\frac{\pi}{4}\right)\left(1+O\left(r^{\alpha-1}\right)\right)
\end{aligned}
$$

as $r \rightarrow 0$, where non-zero constant $p_{m \lambda}$ can be determine exactly. Thus, if $\alpha>1$ and $\lambda>c$, then the functions

$$
P_{m}(r ; \lambda)=P_{m}^{(2)}(R ; \lambda) P_{m}^{(1)}(r ; \lambda)-P_{m}^{(1)}(R ; \lambda) P_{m}^{(2)}(r ; \lambda)
$$

represent the eigenfunctions of $S L$-problem (9), (10), and, consequently,

$$
P_{m}(r ; \lambda) \times \begin{cases}\cos m \varphi, & m \in \mathbb{N}_{0} \\ \sin m \varphi, & m \in \mathbb{N}\end{cases}
$$

are continuous in $\bar{D}$ eigenfunctions of $E V$-problem (7), (8) for each $\lambda \in(c,+\infty)$. Thus, the spectrum of this problem is non-discrete.

If $\alpha=1$, then one can readily see that the eigenfunctions of $S L$-problem (9), (10) are of the shape

$$
\sin \left(\sqrt{\lambda-c-m^{2}} \ln \frac{R}{r}\right), \quad \lambda>c+m^{2} .
$$

In this case, we obtain the following set of eigenfunctions of $E V$-problem (7), (8):

$$
\begin{aligned}
& \sin \left(\sqrt{\lambda-c-m^{2}} \ln \frac{R}{r}\right) \times \begin{cases}\cos m \varphi, & m \in \mathbb{N}_{0} \\
\sin m \varphi, & m \in \mathbb{N}\end{cases} \\
& \lambda \in\left(c+m^{2},+\infty\right) .
\end{aligned}
$$

Thus, we have also continuous spectrum of $E V$-problem (7), (8). 


\section{Expansion of functions by the eigenfunctions of $E V$-problem}

We shall deal with the conditions under which a continuous function $g(r, \varphi)$, having pe$\operatorname{riod} 2 \pi$ with respect to $\varphi$, can be expressed in the eigenfunctions (13), (16) or (17) of $E V$ problem (7), (8).

Let $g$ and $\frac{\partial g}{\partial \varphi} \in C(\bar{D})$. Then one can expand the function $g(r, \varphi)$ by uniformly and absolutely convergence in the $\bar{D}$ Fourier series [12]

$$
g(r, \varphi)=\frac{1}{2} a_{0}(r)+\sum_{m=1}^{\infty}\left(a_{m}(r) \cos m \varphi+b_{m}(r) \sin m \varphi\right),
$$

where

$$
\left.\begin{array}{l}
a_{m}(r) \\
b_{m}(r)
\end{array}\right\}=\frac{1}{\pi} \int_{-\pi}^{\pi} g(r, \varphi)\left\{\begin{array}{c}
\cos m \varphi \\
\sin m \varphi
\end{array}\right\} \mathrm{d} \varphi, \quad \begin{array}{ll}
m \in \mathbb{N}_{0} \\
m \in \mathbb{N} .
\end{array}
$$

Note that, if we want have the expansion of the function $g(r, \varphi)$ by eigenfunctions of $E V$ problem, it is sufficient to expand the coefficients $a_{m}(r)$ and $b_{m}(r)$ of the series (18) in the eigenfunctions of $S L$-problem (9), (10).

Lemma 1 Let $\alpha<1$. Assume that

$$
g \text { and } \frac{\partial g}{\partial \varphi} \in C(\bar{D}), \quad \frac{\partial g}{\partial r} \in C\left(D_{0} \cup K\right), \quad g(R, \varphi)=0 \quad \forall \varphi \in[-\pi, \pi],
$$

and

$$
\int_{0}^{R}\left|\frac{\partial g(r, \varphi)}{\partial r}\right| \mathrm{d} r<\infty \quad \forall \varphi \in[-\pi, \pi] .
$$

Then the functions $a_{m}(r)$ and $b_{m}(r)$ can be expanded by the Fourier-Bessel series:

$$
\left.\begin{array}{l}
a_{m}(r) \\
b_{m}(r)
\end{array}\right\}=\sum_{n=0}^{\infty}\left\{\begin{array}{l}
a_{m n} \\
b_{m n}
\end{array}\right\} J_{\frac{m}{1-\alpha}}\left(\gamma_{m n}\left(\frac{r}{R}\right)^{1-\alpha}\right), \quad \begin{aligned}
& m \in \mathbb{N}_{0} \\
& m \in \mathbb{N}
\end{aligned}
$$

where

$$
\begin{aligned}
\left.\begin{array}{c}
a_{m n} \\
b_{m n}
\end{array}\right\}= & \frac{2(1-\alpha) R^{2(\alpha-1)}}{\pi J_{\frac{m}{1-\alpha}+1}^{2}\left(\gamma_{m n}\right)} \\
& \times \int_{0}^{R} J_{\frac{m}{1-\alpha}}\left(\gamma_{m n}\left(\frac{r}{R}\right)^{1-\alpha}\right) r^{1-2 \alpha} \mathrm{d} r \int_{-\pi}^{\pi} g(r, \varphi)\left\{\begin{array}{c}
\cos m \varphi \\
\sin m \varphi
\end{array}\right\} \mathrm{d} \varphi .
\end{aligned}
$$

These series converge uniformly and absolutely on each interval $[\delta, R], 0<\delta<R$.

If, besides (20),

$$
g(0, \varphi)=0 \quad \forall \varphi \in[-\pi, \pi], \quad \frac{\partial^{2} g}{\partial r^{2}} \in C\left(D_{0} \cup K\right)
$$

and, in addition,

$$
\frac{\partial g(r, \varphi)}{\partial r}=O\left(r^{1-2 \alpha}\right), \quad \frac{\partial^{2} g(r, \varphi)}{\partial r^{2}}=O\left(r^{-2 \alpha}\right) \quad \text { as } r \rightarrow 0
$$


uniformly with respect to $\varphi$, then the series (21) converges uniformly and absolutely on the interval $[0, R]$.

Proof We prove representation (21) for the function $a_{m}(r)$. (This presentation for function $b_{m}(r)$ can be proved analogously.)

By the change of variables $t=\left(\frac{r}{R}\right)^{1-\alpha}$, we introduce the function

$$
\tilde{a}_{m}(t):=a_{m}\left(R t^{\frac{1}{1-\alpha}}\right) .
$$

Then, by virtue of (19),

$$
\tilde{a}_{m}(t)=\frac{1}{\pi} \int_{-\pi}^{\pi} g\left(R t^{\frac{1}{1-\alpha}}, \varphi\right) \cos m \varphi \mathrm{d} \varphi=\frac{1}{\pi} \int_{-\pi}^{\pi} g(r, \varphi) \cos m \varphi \mathrm{d} \varphi
$$

consequently,

$$
\frac{\mathrm{d} \tilde{a}_{m}(t)}{\mathrm{d} t}=\frac{R^{1-\alpha}}{\pi(1-\alpha)} r^{\alpha} \int_{-\pi}^{\pi} \frac{\partial g(r, \varphi)}{\partial r} \cos m \varphi \mathrm{d} \varphi .
$$

Note that $\tilde{a}_{m}(1)=0$ and the function $\tilde{a}_{m}(t)$ is continuous on the interval $[0,1]$ (because of (20)), and

$$
\begin{aligned}
\int_{0}^{1}\left|\frac{\mathrm{d} \tilde{a}_{m}(t)}{\mathrm{d} t}\right| \mathrm{d} t & =\frac{1}{\pi} \int_{0}^{R}\left|\int_{-\pi}^{\pi} \frac{\partial g(r, \varphi)}{\partial r} \cos m \varphi \mathrm{d} \varphi\right| \mathrm{d} r \\
& \leq \frac{1}{\pi} \int_{-\pi}^{\pi} \mathrm{d} \varphi \int_{0}^{R}\left|\frac{\partial g(r, \varphi)}{\partial r}\right| \mathrm{d} r<\infty
\end{aligned}
$$

(by virtue of (20a)). These properties of the function $\tilde{a}_{m}(t)$ are sufficient in order to expand it on the each interval $(\tilde{\delta}, 1], 0<\tilde{\delta}<1$, into a uniformly converging Fourier-Bessel series (see [10], p.615, or [12], p.231, Theorem 2):

$$
\tilde{a}_{m}(t)=\sum_{n=0}^{\infty} a_{m n} J_{\frac{m}{1-\alpha}}\left(\gamma_{m n} t\right), \quad m \in \mathbb{N}_{0},
$$

where

$$
\begin{aligned}
a_{m n} & =\frac{2}{J_{\frac{m}{1-\alpha}+1}^{2}\left(\gamma_{m n}\right)} \int_{0}^{1} \tilde{a}_{m}(t) J_{\frac{m}{1-\alpha}}\left(\gamma_{m n} t\right) t \mathrm{~d} t \\
& =\frac{2}{\pi J_{\frac{m}{1-\alpha}+1}^{2}\left(\gamma_{m n}\right)} \int_{0}^{1} J_{\frac{m}{1-\alpha}}\left(\gamma_{m n} t\right) t \mathrm{~d} t \int_{-\pi}^{\pi} g\left(R t^{\frac{1}{1-\alpha}}, \varphi\right) \cos m \varphi \mathrm{d} \varphi .
\end{aligned}
$$

Substituting into the obtained expressions $t=\left(\frac{r}{R}\right)^{1-\alpha}$, by straightforward calculations, we get under conditions (20), (20a) the representation of the function $a_{m}(r)$ in the FourierBessel series (21) uniformly and absolutely converging on the interval $(\delta, R], \delta=R \tilde{\delta} \frac{1}{1-\alpha}$.

Let, in addition to (20), conditions (23) and (23a) hold. (Note that the first of conditions (23a) implies (20a).) Then

$$
\tilde{a}_{m}(0)=0, \quad \frac{\mathrm{d} \tilde{a}_{m}(t)}{\mathrm{d} t}=O(t) \quad \text { as } t \rightarrow 0,
$$




$$
\begin{aligned}
\frac{\mathrm{d}^{2} \tilde{a}_{m}(t)}{\mathrm{d} t^{2}}= & \frac{R^{2(1-\alpha)}}{(1-\alpha)^{2}} r^{2 \alpha} \int_{-\pi}^{\pi} \frac{\partial^{2} g(r, \varphi)}{\partial r^{2}} \cos m \varphi \mathrm{d} \varphi \\
& +\frac{\alpha R^{2(1-\alpha)}}{(1-\alpha)^{2}} r^{2 \alpha-1} \int_{-\pi}^{\pi} \frac{\partial g(r, \varphi)}{\partial r} \cos m \varphi \mathrm{d} \varphi=O(1) \quad \text { as } t \rightarrow 0,
\end{aligned}
$$

i.e.,

$$
\tilde{a}_{m}(0)=0,\left.\quad \frac{\mathrm{d} \tilde{a}_{m}(t)}{\mathrm{d} t}\right|_{t=0}=0, \quad\left|\frac{\mathrm{d}^{2} \tilde{a}_{m}(t)}{\mathrm{d} t^{2}}\right|<\infty, \quad 0<t \leq 1 .
$$

These additional conditions yield the uniformly and absolutely convergence of the series (24) on the interval [0,1] (see [12], p.222, Theorem 3). Consequently, the series (21) of the function $a_{m}(r)$ converges uniformly and absolutely on the interval $[0, R]$.

Lemma 2 Let $\alpha>1$. Assume that conditions (20), (20a) of Lemma 1 are satisfied and

$$
\int_{0}^{R} r^{\frac{1-3 \alpha}{2}} \mathrm{~d} r \int_{-\pi}^{\pi}|g(r, \varphi)| \mathrm{d} \varphi<\infty .
$$

Then the functions $a_{m}(r)$ and $b_{m}(r)$ can be represented on the interval $[0, R]$ by the following uniformly convergent integrals:

$$
\left.\begin{array}{l}
a_{m}(r) \\
b_{m}(r)
\end{array}\right\}=\int_{c}^{\infty}\left\{\begin{array}{l}
A_{m}(\lambda) \\
B_{m}(\lambda)
\end{array}\right\} P_{m}(r ; \lambda) \mathrm{d} \lambda, \quad \begin{aligned}
& m \in \mathbb{N}_{0} \\
& m \in \mathbb{N}
\end{aligned}
$$

where

$$
\begin{aligned}
& \left.\begin{array}{l}
A_{m}(\lambda) \\
B_{m}(\lambda)
\end{array}\right\}=\frac{1}{2} \int_{c}^{\infty} \frac{1}{\pi(\alpha-1)\left(P_{m}^{(1)}(R ; \lambda)\right)^{2}+\left(P_{m}^{(2)}(R ; \lambda)\right)^{2}} \\
& \times \int_{0}^{R} P_{m}(r ; \lambda) r^{1-2 \alpha} \mathrm{d} r \int_{-\pi}^{\pi}\left\{\begin{array}{cc}
\cos m \varphi \\
\sin m \varphi
\end{array}\right\} g(r, \varphi) \mathrm{d} \varphi, \quad \begin{array}{l}
m \in \mathbb{N}_{0}, \\
m \in \mathbb{N} .
\end{array}
\end{aligned}
$$

(Here functions $P_{m}^{(1)}, P_{m}^{(2)}$ and $P_{m}$ are defined by (14) and (15).)

Proof Likewise as in Lemma 1, we prove representation (27) only for the function $a_{m}(r)$.

Denote

$$
t=\frac{r^{1-\alpha}}{\alpha-1}, \quad t_{R}=\frac{R^{1-\alpha}}{\alpha-1}
$$

and introduce the function $a_{m}^{*}(t):=a_{m}\left(((\alpha-1) t)^{\frac{1}{1-\alpha}}\right)$. Then

$$
\begin{aligned}
& a_{m}^{*}(t)=\frac{1}{\pi} \int_{-\pi}^{\pi} g\left(\left(\frac{t}{\alpha-1}\right)^{\frac{1}{1-\alpha}}, \varphi\right) \cos m \varphi \mathrm{d} \varphi, \quad t_{R} \leq t<+\infty, \\
& \frac{\mathrm{d} \tilde{a}_{m}(t)}{\mathrm{d} t}=-\frac{1}{\pi} r^{\alpha} \int_{-\pi}^{\pi} \frac{\partial g(r, \varphi)}{\partial r} \cos m \varphi \mathrm{d} \varphi,
\end{aligned}
$$

and

$$
\int_{t_{R}}^{\infty}\left|\frac{\mathrm{d} a_{m}^{*}(t)}{\mathrm{d} t}\right| \mathrm{d} t=\frac{1}{\pi} \int_{0}^{R}\left|\int_{-\pi}^{\pi} \frac{\partial g(r, \varphi)}{\partial r} \cos m \varphi \mathrm{d} \varphi\right| \mathrm{d} r \leq \frac{1}{\pi} \int_{-\pi}^{\pi} \mathrm{d} \varphi \int_{0}^{R}\left|\frac{\partial g(r, \varphi)}{\partial r}\right| \mathrm{d} r<\infty
$$


due to the condition of (20a). Since $a_{m}^{*}\left(t_{R}\right)=a_{m}(R)=0$ (because of $g(R, \varphi)=0$ ),

$$
\begin{aligned}
\int_{t_{R}}^{\infty} \sqrt{t}\left|a_{m}^{*}(t)\right| \mathrm{d} t & =\frac{1}{\sqrt{\alpha-1}} \int_{0}^{R} r^{\frac{1-3 \alpha}{2}}\left|a_{m}(r)\right| \mathrm{d} r \\
& =\frac{1}{\pi \sqrt{\alpha-1}} \int_{0}^{R} r^{\frac{1-3 \alpha}{2}}\left|\int_{-\pi}^{\pi} g(r, \varphi) \cos m \varphi \mathrm{d} \varphi\right| \mathrm{d} r \\
& \leq \frac{1}{\pi} \int_{0}^{R} r^{\frac{1-3 \alpha}{2}} \mathrm{~d} r \int_{-\pi}^{\pi}|g(r, \varphi)| \mathrm{d} \varphi<\infty
\end{aligned}
$$

(by virtue of condition of (26)), the function $a_{m}^{*}(t)$ can be expanded on the interval $\left[t_{R}, \infty\right)$ by a uniformly convergent Weber-Orr integral (see [13], p.74),

$$
a_{m}^{*}(t)=\int_{0}^{\infty} A_{m}^{*}(\mu)\left(N_{\frac{m}{\alpha-1}}\left(\mu t_{R}\right) J_{\frac{m}{\alpha-1}}(\mu t)-J_{\frac{m}{\alpha-1}}\left(\mu t_{R}\right) N_{\frac{m}{\alpha-1}}(\mu t)\right) \mu \mathrm{d} \mu,
$$

where

$$
\begin{aligned}
A_{m}^{*}(\mu)= & \frac{1}{\left.2 \pi(\alpha-1) J_{\frac{m}{\alpha-1}}^{2}\left(\mu t_{R}\right)+N_{\frac{m}{\alpha-1}}^{2}\left(\mu t_{R}\right)\right)} \\
& \times \int_{t_{R}}^{\infty} a_{m}^{*}(t)\left(N_{\frac{m}{\alpha-1}}\left(\mu t_{R}\right) J_{\frac{m}{\alpha-1}}(\mu t)-J_{\frac{m}{\alpha-1}}\left(\mu t_{R}\right) N_{\frac{m}{\alpha-1}}(\mu t)\right) t \mathrm{~d} t .
\end{aligned}
$$

Therefore, substituting $\mu=\sqrt{\lambda-c}, \lambda \in(c,+\infty)$, in the expressions of the functions $a_{m}^{*}(t)$ and $A_{m}^{*}(\mu)$ obtained above, whereupon denoting

$$
A_{m}(\lambda)=\frac{1}{2} A_{m}^{*}(\sqrt{\lambda-c})
$$

and taking into account both definitions (14) and (15), we obtain

$$
a_{m}(r)=\int_{c}^{\infty} A_{m}(\lambda) P_{m}(r ; \lambda) \mathrm{d} \lambda, \quad r \in[0, R]
$$

where

$$
\begin{aligned}
A_{m}(\lambda)= & \frac{1}{\pi(\alpha-1)\left(P_{m}^{(1)}(R ; \lambda)\right)^{2}+\left(P_{m}^{(2)}(R ; \lambda)\right)^{2}} \\
& \times \int_{0}^{R} a_{m}(r) P_{m}(r ; \lambda) r^{1-2 \alpha} \mathrm{d} r, \quad m \in \mathbb{N}_{0} .
\end{aligned}
$$

Putting equation (19) of the functions $a_{m}(r)$ into the last equality we get equation (27).

Presentation (27) for the function $b_{m}(r)$ is obtained analogously.

Lemma 3 Let $\alpha=1$ and let the assumptions of Lemma 2 hold. Then functions $a_{m}(r)$ and $b_{m}(r)$ integrals uniformly convergent on the each interval $[\delta, R], 0<\delta<R$ can be represented by the following:

$$
\left.\begin{array}{l}
a_{m}(r) \\
b_{m}(r)
\end{array}\right\}=\int_{c+m^{2}}^{\infty}\left\{\begin{array}{l}
\alpha_{m}(\lambda) \\
\beta_{m}(\lambda)
\end{array}\right\} \sin \left(\sqrt{\lambda-c-m^{2}} \ln \frac{R}{r}\right) \mathrm{d} \lambda, \quad \begin{aligned}
& m \in \mathbb{N}_{0} \\
& m \in \mathbb{N}
\end{aligned}
$$




$$
\left.\begin{array}{c}
\alpha_{m}(\lambda) \\
\beta_{m}(\lambda)
\end{array}\right\}=\frac{1}{\pi^{2} \sqrt{\lambda-c-m^{2}}} \int_{0}^{R} \sin \left(\sqrt{\lambda-c-m^{2}} \ln \frac{R}{r}\right) \frac{\mathrm{d} r}{r} \int_{-\pi}^{\pi} g(r, \varphi)\left\{\begin{array}{l}
\cos m \varphi \\
\sin m \varphi
\end{array}\right\} \mathrm{d} \varphi .
$$

Proof By the change of variable $t=\ln \frac{r}{R}$, we introduce the functions $\hat{a}_{m}(t):=a_{m}\left(R e^{t}\right)$. It is easily seen that $\hat{a}_{m}(0)=a_{m}(R)=0$. Further, just repeating the reasoning of Lemma 2 , we show that

$$
\int_{0}^{\infty}\left|\hat{a}_{m}(t)\right| \mathrm{d} t<\infty, \quad \int_{0}^{\infty}\left|\frac{\mathrm{d} \hat{a}_{m}(t)}{\mathrm{d} t}\right| \mathrm{d} t<\infty
$$

Hence, we have the Fourier sine-expansion (see [12], p.190, or [14], p.71)

$$
\hat{a}_{m}(t)=\int_{0}^{\infty} \hat{A}_{m}(\mu) \sin \mu t \mathrm{~d} \mu, \quad t \in[0,+\infty),
$$

where

$$
\hat{A}_{m}(\mu)=\frac{2}{\pi} \int_{0}^{\infty} \hat{a}_{m}(t) \sin \mu t \mathrm{~d} t
$$

Then, by the substitution

$$
\mu=\gamma_{m}(\lambda):=\sqrt{\lambda-c-m^{2}}, \quad \lambda \in\left(c+m^{2},+\infty\right),
$$

we obtain

$$
\begin{aligned}
& a_{m}(r)=\hat{a}_{m}\left(\ln \frac{R}{r}\right)=\frac{1}{2} \int_{c+m^{2}}^{\infty} \frac{\hat{A}_{m}\left(\gamma_{m}(\lambda)\right)}{\gamma_{m}(\lambda)} \sin \left(\gamma_{m}(\lambda) \ln \frac{r}{R}\right) \mathrm{d} \lambda, \quad r \in(0, R], \\
& \hat{A}_{m}\left(\gamma_{m}(\lambda)\right)=\frac{2}{\pi} \int_{0}^{R} a_{m}(r) \sin \left(\gamma_{m}(\lambda) \ln \frac{r}{R}\right) \frac{\mathrm{d} r}{r} .
\end{aligned}
$$

Thus, taking into account equalities (20) and denoting

$$
\alpha_{m}(\lambda)=\frac{\tilde{A}_{m}\left(\gamma_{m}(\lambda)\right)}{\pi^{2} \gamma_{m}(\lambda)}
$$

we get expression (29).

Presentation (28) for function $b_{m}(r)$ is obtained analogously.

\section{Solutions of Problem D1}

Let $Z(z ; \lambda)$ be any solution of the differential equation

$$
Z^{\prime \prime}-\lambda Z=0, \quad \lambda \in \mathbb{R},
$$

and let $W(r, \varphi ; \lambda)$ be any solution of the $E V$-problem. Then $U(r, \varphi, z ; \lambda)=W(r, \varphi ; \lambda) Z(z ; \lambda)$ is the partial solution of equation (2), which is bounded in $Q_{0}$ such that $U(R, \varphi, z ; \lambda)=0$. We shall get the presentation of the solution of Problem D1 as some composition of those partial solutions. 
Lemma 4 The bounded in $Q_{0}$ solutions of equation (2) can attain either a positive maximum or a negative minimum only on the boundary $\partial Q$ of cylinder $Q$.

Proof Due to the ellipticity of equation (2) in $Q_{0}$ and in view of condition $c \geq 0$, the solutions of this equation cannot attain in $Q_{0}$ neither a positive maximum nor a negative minimum $[15,16]$. Hence, it suffices to prove that bounded solutions cannot attain an extremum on the line of degeneracy $r=0,0<z<H$.

Let $u$ be the solution of equation (2) bounded on the line $r=0$ and let $v(\varepsilon)$ be its positive maximum on the surface $\{r=\varepsilon, 0<z<H\}$. Denote this maximum point by $M_{\varepsilon}$. Note that $v(\varepsilon)$ monotonically decreases with respect to $\varepsilon$. Since $u_{z z}$ and $v_{\varphi \varphi}$ are non-positive at the maximum point, we get from equation (2)

$$
\frac{\partial u}{\partial r}\left(r u_{r}\right) \geq 0
$$

and, due to the Zaremba-Giraud [17] principle, $u_{r}<0$ at the point $M_{\varepsilon}$.

Denote $\omega(\varepsilon)=\varepsilon u_{r}\left(M_{\varepsilon}\right)$. It follows from (31) that $\omega(\varepsilon)$ is a negative monotonically increasing function. Thus, there exists a constant $k<0$ such that $\omega(\varepsilon) \leq k$ for small enough $\varepsilon$. Note that the function $v(\varepsilon)$ is continuously differentiable on the interval $(0, R)$ because of the ellipticity of equation (2) in $Q_{0}$. Therefore $v^{\prime}(\varepsilon)=u_{r}\left(M_{\varepsilon}\right)=\varepsilon^{-1} \omega(\varepsilon)$, and we obtain

$$
v^{\prime}(\varepsilon) \leq k \varepsilon^{-1},
$$

if $\varepsilon$ is small enough. The integration of this inequality on the interval $\left(\varepsilon, r_{0}\right)$, where $r_{0}$ is small enough, yields the estimate

$$
v(\varepsilon) \geq \text { const }+k \ln \varepsilon .
$$

Hence, $v(\varepsilon) \rightarrow \infty$, as $\varepsilon \rightarrow 0$, i.e. the solution $u$ is not bounded on the line $r=0$, but that is in contradiction with the postulate of the lemma.

One can prove, analogously, that $u$ cannot attain any negative minimum on the line $r=0$, $0<z<H$.

Let the functions $f_{i}(r, \varphi), i=1,2$, from (5) be such that $\frac{\partial f_{i}}{\partial \varphi} \in C(\bar{D})$. Then, as is mentioned above, these functions can be expanded by uniformly and absolutely converging in the $\bar{D}$ Fourier series,

$$
f_{i}(r, \varphi)=\frac{1}{2} a_{0}^{(i)}(r)+\sum_{m=1}^{\infty}\left(a_{m}^{(i)}(r) \cos m \varphi+b_{m}^{(i)}(r) \sin m \varphi\right),
$$

where

$$
\left.\begin{array}{l}
a_{m}^{(i)}(r) \\
b_{m}^{(i)}(r)
\end{array}\right\}=\frac{1}{\pi} \int_{-\pi}^{\pi} f_{i}(r, \varphi)\left\{\begin{array}{c}
\cos m \varphi \\
\sin m \varphi
\end{array}\right\} \mathrm{d} \varphi, \quad \begin{aligned}
& m \in \mathbb{N}_{0} \\
& m \in \mathbb{N} .
\end{aligned}
$$

I. Let $\alpha<1$. Assume that both functions $f_{i}$ satisfy conditions (20), (20a) of Lemma 1 . Then, according to this lemma, the coefficients $a_{m}^{(i)}(r)$ and $b_{m}^{(i)}(r)$ of the series (32) can be 
expanded by uniformly converging in each ring $\bar{D}_{\delta}, 0<\delta<R$, the Fourier-Bessel series of shape (21), i.e., the representations

$$
\begin{aligned}
f_{i}(r, \varphi)= & \frac{1}{2} \sum_{n=0}^{\infty} a_{0 n}^{(i)} J_{0}\left(\gamma_{0 n}\left(\frac{r}{R}\right)^{1-\alpha}\right) \\
& +\sum_{m=1}^{\infty} \sum_{n=0}^{\infty} J_{\frac{m}{1-\alpha}}\left(\gamma_{m n}\left(\frac{r}{R}\right)^{1-\alpha}\right)\left(a_{m n}^{(i)} \cos m \varphi+b_{m n}^{(i)} \sin m \varphi\right), \quad i=1,2,
\end{aligned}
$$

with the coefficients $a_{m n}^{(i)}, b_{m n}^{(i)}$ defined analogously to (22) hold.

Choose the values $\lambda_{m n}$ of parameter $\lambda$ in equation (30) by equation (11). Let $Z_{m n}^{(i)}(z)$, $i=1,2$, be the solutions of the equation

$$
Z^{\prime \prime}-\lambda_{m n} Z=0, \quad m, n \in \mathbb{N}_{0}
$$

satisfying the boundary value conditions

$$
Z_{m n}^{(1)}(0)=a_{m n}^{(1)}, \quad Z_{m n}^{(1)}(H)=a_{m n}^{(2)} ; \quad Z_{m n}^{(2)}(0)=b_{m n}^{(1)}, \quad Z_{m n}^{(2)}(H)=b_{m n}^{(2)} .
$$

It is easily seen that those solutions are as follows:

$$
\begin{aligned}
& Z_{m n}^{(1)}(z)=\sinh ^{-1} \sqrt{\lambda_{m n}} H\left(a_{m n}^{(1)} \sinh \sqrt{\lambda_{m n}}(H-z)+a_{m n}^{(2)} \sinh \sqrt{\lambda_{m n}} z\right), \\
& Z_{m n}^{(2)}(z)=\sinh ^{-1} \sqrt{\lambda_{m n}} H\left(b_{m n}^{(1)} \sinh \sqrt{\lambda_{m n}}(H-z)+b_{m n}^{(2)} \sinh \sqrt{\lambda_{m n}} z\right) .
\end{aligned}
$$

So we obtain the sequence

$$
J_{\frac{m}{1-\alpha}}\left(\gamma_{m n}\left(\frac{r}{R}\right)^{1-\alpha}\right) \times\left\{\begin{array}{l}
Z_{m n}^{(1)}(z) \cos m \varphi, \\
Z_{m n}^{(2)}(z) \sin m \varphi,
\end{array} \quad m, n \in \mathbb{N}_{0},\right.
$$

of partial solutions of equation (2), which are continuous in $\bar{Q}$.

Let us compose the series

$$
\begin{aligned}
u_{1}(r, \varphi, z)= & \frac{1}{2} \sum_{n=0}^{\infty} J_{0}\left(\gamma_{0 n}\left(\frac{r}{R}\right)^{1-\alpha}\right) Z_{0 n}^{(1)}(z) \\
& +\sum_{m=1}^{\infty} \sum_{n=0}^{\infty} J_{\frac{m}{1-\alpha}}\left(\gamma_{m n}\left(\frac{r}{R}\right)^{1-\alpha}\right)\left(Z_{m n}^{(1)}(z) \cos m \varphi+Z_{m n}^{(2)}(z) \sin m \varphi\right) .
\end{aligned}
$$

If $z=0$ and $z=H$, then this series coincides with the series (33) of functions $f_{1}$ and $f_{2}$, obviously:

$$
u_{1}(r, \varphi,(i-1) H)=f_{i}(r, \varphi) \quad \forall(r, \varphi) \in \bar{D}_{\delta}, i=1,2 .
$$

Therefore, the series (34) converges uniformly on the two bases of cylinder $Q$ except, maybe, the points $(r=0, z=(i-1) H), i=1,2$, and also on the lateral surface of this cylinder (by virtue of $\left.J_{\frac{m}{1-\alpha}}\left(\gamma_{m n}\right)=0, m, n \in \mathbb{N}_{0}\right)$. Since equation (2) is elliptic in domain $Q_{0}$, this jointly with Lemma 4 yields the uniform convergence of this series everywhere in each 
domain $\bar{Q}_{\delta}, 0<\delta<R$, because of the maximum principle for elliptic equations. Moreover, the sum $u_{1}(r, \varphi, z)$ of the series (34) represents the solution of equation (2) from the class $C^{2}\left(Q_{0}\right) \cap C(\bar{Q} \backslash\{r=0\})$ and satisfies the boundary value conditions (3), (4).

If functions $f_{i}, i=1,2$, satisfy not only condition (20) but also conditions (23), (23a), then, according to Lemma 1 , the series (33) converges uniformly and absolutely in the disk $\bar{D}$. Then it follows from (34) that

$$
u_{1}(0, \varphi, z)=\frac{1}{2} \sum_{n=0}^{\infty} Z_{0 n}^{(1)}(z)=\frac{1}{2} \sum_{n=0}^{\infty} \frac{a_{0 n}^{(1)} \sinh \sqrt{\lambda_{0 n}}(H-z)+a_{0 n}^{(2)} \sinh \sqrt{\lambda_{0 n} z}}{\sinh \sqrt{\lambda_{0 n}} H} ;
$$

consequently,

$$
\left|u_{1}(0, \varphi, z)\right| \leq \frac{1}{2} \sum_{n=0}^{\infty}\left(\left|a_{0 n}^{(1)}\right|+\left|a_{0 n}^{(2)}\right|\right)<\infty, \quad 0 \leq z \leq H,
$$

because of the absolute convergence of the series (33) in $\bar{D}$. Thus, the series (34) converges uniformly everywhere in $\bar{D}$ (including the line of degeneracy $r=0$ ), i.e., $u_{1}$ is the solution of Problem D1 from the class $C^{2}\left(Q_{0}\right) \cap C(\bar{Q})$.

Besides, due to Lemma 4 and to the ellipticity of equation (2), solution $u_{1}$ of Problem D1 can attain the positive maximum or negative minimum only on the bases of cylinder $Q$. This yields the estimate

$$
\left|u_{1}(r, \varphi, z)\right|<\max \left\{\max _{\bar{D}}\left|f_{1}(r, \varphi)\right|, \max _{\bar{D}}\left|f_{2}(r, \varphi)\right|\right\}, \quad(r, \varphi, z) \in Q_{0},
$$

which implies the uniqueness of the solution Problem D1.

Thus, the following theorem holds.

Theorem 1 Let $\alpha<1$. Iff $f_{i}$ and $\frac{\partial f_{i}}{\partial \varphi} \in C(\bar{D}), \frac{\partial f_{i}}{\partial r} \in C\left(D_{0} \cup K\right), f_{i}(R, \varphi)=0 \forall \varphi \in[-\pi, \pi]$ and

$$
\int_{0}^{R}\left|\frac{\partial f_{i}(r, \varphi)}{\partial r}\right| \mathrm{d} r<\infty \quad \forall \varphi \in[-\pi, \pi], i=1,2
$$

then Problem D1 has the unique solution $u_{1} \in C^{2}\left(Q_{0}\right) \cap C(\bar{Q} \backslash\{r=0\})$, which can be represented by (34).

If, in addition, $\frac{\partial^{2} f_{i}}{\partial r^{2}} \in C\left(D_{0} \cup K\right), f_{i}(0, \varphi)=0, \forall \varphi \in[-\pi, \pi]$, and

$$
\frac{\partial f_{i}(r, \varphi)}{\partial r}=O\left(r^{1-2 \alpha}\right), \quad \frac{\partial^{2} f_{i}(r, \varphi)}{\partial r^{2}}=O\left(r^{-2 \alpha}\right), \quad i=1,2
$$

as $r \rightarrow 0$, uniformly with respect to $\varphi$, then the series (34) represents the solution of Problem $\mathrm{D} 1$ from the class $C^{2}\left(Q_{0}\right) \cap C(\bar{Q})$.

II. Assume that $\alpha>1$. Let the assumptions of Lemma 2 be satisfied. According to this lemma, the functions can be presented on the interval $[0, R]$ by the series $(32)$ with the coefficients $a_{m}^{(i)}(r)$ and $b_{m}^{(i)}(r)$ of shape (27):

$$
\left.\begin{array}{l}
a_{m}^{(i)}(r) \\
b_{m}^{(i)}(r)
\end{array}\right\}=\int_{c}^{\infty}\left\{\begin{array}{l}
A_{m}^{(i)}(\lambda) \\
B_{m}^{(i)}(\lambda)
\end{array}\right\} P_{m}(r ; \lambda) \mathrm{d} \lambda, \quad \begin{aligned}
& m \in \mathbb{N}_{0} \\
& m \in \mathbb{N}
\end{aligned}
$$


where functions $A_{m}^{(i)}(\lambda)$ and $B_{m}^{(i)}(\lambda)$ are defined by equation (28) applying it to the functions $f_{i}, i=1,2$, respectively.

Introduce the solutions

$$
\begin{aligned}
& Z_{m}^{(1)}(z ; \lambda)=\sinh ^{-1} \sqrt{\lambda} H\left(A_{m}^{(1)}(\lambda) \sinh \sqrt{\lambda}(H-z)+A_{m}^{(2)}(\lambda) \sinh \sqrt{\lambda} z\right), \\
& Z_{m}^{(2)}(z ; \lambda)=\sinh ^{-1} \sqrt{\lambda} H\left(B_{m}^{(1)}(\lambda) \sinh \sqrt{\lambda}(H-z)+B_{m}^{(2)}(\lambda) \sinh \sqrt{\lambda} z\right),
\end{aligned}
$$

of equation (30), which satisfy the obvious conditions:

$$
\begin{array}{ll}
Z_{m}^{(1)}(0 ; \lambda)=A_{m}^{(1)}(\lambda), & Z_{m}^{(1)}(H ; \lambda)=A_{m}^{(2)}(\lambda), \\
Z_{m}^{(2)}(0 ; \lambda)=B_{m}^{(1)}(\lambda), & Z_{m}^{(2)}(H ; \lambda)=B_{m}^{(2)}(\lambda) .
\end{array}
$$

So we obtain the set

$$
P_{m}(r ; \lambda) \times\left\{\begin{array}{ll}
Z_{m}^{(1)}(z ; \lambda) \cos m \varphi, & m \in \mathbb{N}_{0}, \\
Z_{m}^{(2)}(z ; \lambda) \sin m \varphi, & m \in \mathbb{N},
\end{array} \quad \lambda \in(c,+\infty),\right.
$$

of the partial solutions of equation (2), which are continuous in $\bar{Q}$.

Let us consider the integrals

$$
I_{m}^{(i)}(r, z)=\int_{c}^{\infty} Z_{m}^{(i)}(z ; \lambda) P_{m}(r ; \lambda) \mathrm{d} \lambda, \quad(r, z) \in S, i=1,2 .
$$

We shall prove the uniform convergence of these integrals in the domain $\bar{S}$. We confine oneself to the case $i=1$. Introduce the function

$$
\tilde{A}_{m}^{(1)}(r, z ; l)=\int_{c}^{l} Z_{m}^{(1)}(z ; \lambda) P_{m}(r ; \lambda) \mathrm{d} \lambda,
$$

where $l \in(c,+\infty)$. One can check by direct calculation that the function $\tilde{A}_{m}^{(1)}(r, z ; l)$ satisfy the equation

$$
W_{z z}+r^{2 \alpha}\left(W_{r r}+\frac{1}{r} W_{r}\right)-\left(m^{2} r^{2(\alpha-1)}+c\right) W=0 .
$$

According to the maximum principle, the solutions of this equation can attain a positive maximum or negative minimum only on the boundary of domain $S$. Since

$$
\tilde{A}_{m}^{(1)}(R, \varphi, z ; l)=\tilde{A}_{m}^{(1)}(0, \varphi, z ; l)=0
$$

(in view of $\left.P_{m}(R ; \lambda)=P_{m}(0 ; \lambda)=0\right)$ and

$$
\tilde{A}_{m}^{(1)}(r,(i-1) H ; l)=\int_{c}^{l} A_{m}^{(i)}(\lambda) P_{m}(r ; \lambda) \mathrm{d} \lambda, \quad i=1,2
$$

(due to (37)), we have the estimate

$$
\left|\tilde{A}_{m}^{(1)}(r, z ; l)\right| \leq \max \left\{\left|\tilde{A}_{m}^{(1)}(r, 0 ; l)\right|,\left|\tilde{A}_{m}^{(1)}(r, H ; l)\right|\right\}
$$


everywhere in $\bar{S}$. Observe that (see (36))

$$
\lim _{l \rightarrow \infty} \tilde{A}_{m}^{(1)}(r,(i-1) H ; l)=\int_{c}^{\infty} A_{m}^{(i)}(\lambda) P_{m}(r ; \lambda) \mathrm{d} \lambda=a_{m}^{(i)}(r), \quad i=1,2 .
$$

Thus, passing to the limit as $l \rightarrow \infty$ in (40), we obtain

$$
\lim _{l \rightarrow \infty}\left|\tilde{A}_{m}^{(1)}(r, z ; l)\right|=\left|\int_{c}^{\infty} Z_{m}^{(1)}(z ; \lambda) P_{m}(r ; \lambda) \mathrm{d} \lambda\right| \leq \max \left\{\left|a_{m}^{(1)}(r)\right|,\left|a_{m}^{(2)}(r)\right|\right\}
$$

in $\bar{S}$, i.e. the integral $I_{m}^{(1)}(r, z)$ converges uniformly in $\bar{S}$.

The uniform convergence of the integral $I_{m}^{(2)}(r, z)$ one can prove analogously.

Note that both functions $I_{m}^{(i)}(r, z), i=1,2$, which can be interpreted as the limit of corresponding functions $\tilde{A}_{m}^{(i)}(r, z ; l)$ as $l \rightarrow \infty$, satisfy equation (39) in $S$ because of the ellipticity of this equation in $S$ and because of the maximum principle.

Let us compose the series,

$$
u_{2}(r, \varphi, z)=\frac{1}{2} I_{0}^{(1)}(r, z)+\sum_{m=1}^{\infty}\left(I_{m}^{(1)}(r, z) \cos m \varphi+I_{m}^{(2)}(r, z) \sin m \varphi\right)
$$

where the functions $I_{m}^{(i)}(r, z), i=1,2$, are defined by (38). Observe that

$$
\nu_{2}(r, \varphi,(i-1) H)=g_{i}(r, \varphi), \quad i=1,2,
$$

because of

$$
\begin{array}{ll}
I_{m}^{(1)}(r, 0)=a_{m}^{(1)}(r), & I_{m}^{(2)}(r, 0)=b_{m}^{(1)}(r), \\
I_{m}^{(1)}(r, H)=a_{m}^{(2)}(r), & I_{m}^{(2)}(r, H)=b_{m}^{(2)}(r),
\end{array}
$$

and $u_{2}(R, \varphi, z)=u_{2}(0, \varphi, z)=0$ (in view of $\left.I_{m}^{(i)}(R ; \lambda)=I_{m}^{(i)}(0 ; \lambda)=0, m=0,1, \ldots\right)$. Thus, the series (41) converges uniformly (and absolutely) on the boundary $\partial Q_{0}$ of cylinder $Q_{0}$. Due to the maximum principle this series converges uniformly in $Q_{0}$ and its sum $u_{2}(r, \varphi, z)$ represents the solution of Problem D1 from the class $C^{2}\left(Q_{0}\right) \cap C(\bar{Q})$. Furthermore, jointly with Lemma 4, the maximum principle yields for the solution $u_{2}$ the same estimate (35) as for the solution $u_{1}$. This implies the uniqueness of the solution $u_{2}$.

The theorem follows from the above reasoning.

Theorem 2 Let $\alpha>1$. Iff $f_{i}$ and $\frac{\partial f_{i}}{\partial \varphi} \in C(\bar{D}), \frac{\partial f_{i}}{\partial r} \in C\left(D_{0} \cup K\right), f_{i}(R, \varphi)=0 \forall \varphi \in[-\pi, \pi], i=1,2$, and the conditions

$$
\int_{0}^{R} r^{\frac{1-3 \alpha}{2}} \mathrm{~d} r \int_{-\pi}^{\pi}\left|f_{i}(r, \varphi)\right| \mathrm{d} \varphi<\infty, \quad \int_{0}^{R}\left|\frac{\partial f_{i}(r, \varphi)}{\partial r}\right| \mathrm{d} r<\infty \quad \forall \varphi \in[-\pi, \pi]
$$

are fulfilled, then Problem D1 has the unique solution $u_{2} \in C^{2}\left(Q_{0}\right) \cap C(\bar{Q})$, which can be represented by (41).

III. Let $\alpha=1$. Assume that functions $f_{i}, i=1,2$, satisfy the conditions of Theorem 2. In this case, according to Lemma 3 , the coefficients $a_{m}^{(i)}(r)$ and $b_{m}^{(i)}(r)$ of the series (32) can be 
presented on the interval $(0, R]$ in the form of integrals of shape (29):

$$
\left.\begin{array}{l}
a_{m}^{(i)}(r) \\
b_{m}^{(i)}(r)
\end{array}\right\}=\int_{c+m^{2}}^{\infty}\left\{\begin{array}{l}
\alpha_{m}^{(i)}(\lambda) \\
\beta_{m}^{(i)}(\lambda)
\end{array}\right\} \sin \left(\sqrt{\lambda-c-m^{2}} \ln \frac{R}{r}\right) \mathrm{d} \lambda, \quad \begin{aligned}
& m \in \mathbb{N}_{0} \\
& m \in \mathbb{N}
\end{aligned}
$$

where functions $\alpha_{m}^{(i)}(\lambda)$ and $\beta_{m}^{(i)}(\lambda)$ are defined analogously to the functions $\alpha_{m}(\lambda)$ and $\beta_{m}(\lambda)$ in (29). Then the series (32) with the coefficients of shape (43) converge uniformly in the each desk $\bar{D}_{\delta}, 0<\delta<R$.

Let us determine the solutions

$$
\begin{aligned}
& \tilde{Z}_{m}^{(1)}(z ; \lambda)=\sinh ^{-1} \sqrt{\lambda} H\left(\alpha_{m}^{(1)}(\lambda) \sinh \sqrt{\lambda}(H-z)+\alpha_{m}^{(2)}(\lambda) \sinh \sqrt{\lambda} z\right), \\
& \tilde{Z}_{m}^{(2)}(z ; \lambda)=\sinh ^{-1} \sqrt{\lambda} H\left(\beta_{m}^{(1)}(\lambda) \sinh \sqrt{\lambda}(H-z)+\beta_{m}^{(2)}(\lambda) \sinh \sqrt{\lambda} z\right),
\end{aligned}
$$

of equation (30) which satisfy the boundary value conditions

$$
\begin{array}{ll}
\tilde{Z}_{m}^{(1)}(0 ; \lambda)=\alpha_{m}^{(1)}(\lambda), & \tilde{Z}_{m}^{(1)}(H ; \lambda)=\alpha_{m}^{(2)}(\lambda), \\
\tilde{Z}_{m}^{(2)}(0 ; \lambda)=\beta_{m}^{(1)}(\lambda), & \tilde{Z}_{m}^{(2)}(H ; \lambda)=\beta_{m}^{(2)}(\lambda),
\end{array}
$$

evidently. Then we get the set

$$
\sin \left(\sqrt{\lambda-c-m^{2}} \ln \frac{R}{r}\right) \times \begin{cases}\tilde{Z}_{m}^{(1)}(z ; \lambda) \cos m \varphi, & m \in \mathbb{N}_{0} \\ \tilde{Z}_{m}^{(2)}(z ; \lambda) \sin m \varphi, & m \in \mathbb{N}\end{cases}
$$

$\lambda \in\left(c+m^{2},+\infty\right)$, of partial solutions of equation (2) which are continuous in each cylindric ring $\bar{Q}_{\delta}$, are bounded in $Q_{0}$, and are equal to zero for $r=R$.

Introduce the integrals

$$
J_{m}^{(i)}(r, z)=\int_{c+m^{2}}^{\infty} \tilde{Z}_{m}^{(i)}(z ; \lambda) \sin \left(\sqrt{\lambda-c-m^{2}} \ln \frac{R}{r}\right) \mathrm{d} \lambda, \quad i=1,2,
$$

and compose the series

$$
u_{3}(r, \varphi, z)=\frac{1}{2} J_{0}^{(1)}(r, z)+\sum_{m=1}^{\infty}\left(J_{m}^{(1)}(r, z) \cos m \varphi+J_{m}^{(2)}(r, z) \sin m \varphi\right) .
$$

The uniform convergence of integrals (44) and possibility of their twice differentiation in $S$ can be proved in the same way as the proof of integrals (38). The difference is only this: since the solutions of problem (9), (10) are non-continuous at the point $r=0$ as $\alpha=1$, the integrals (44) are not defined if $r=0$. (However, these integrals are bounded in $S$.)

It follows from (43) and (44) that

$$
\begin{array}{lll}
J_{m}^{(1)}(r, 0)=a_{m}^{(1)}(r), & J_{m}^{(1)}(r, H)=a_{m}^{(2)}(r), & m \in \mathbb{N}_{0} \\
J_{m}^{(2)}(r, 0)=b_{m}^{(1)}(r), & J_{m}^{(2)}(r, H)=b_{m}^{(2)}(r), & m \in \mathbb{N}
\end{array}
$$

consequently,

$$
u_{3}(r, \varphi,(i-1) H)=f_{i}(r, \varphi) \quad \forall(r, \varphi) \in \bar{D}_{\delta}, i=1,2 .
$$


Moreover, $u_{3}(R, \varphi, z)=0$ because of $J_{m}^{(i)}(R, z) \equiv 0$. Hence the series (45) converges uniformly on $\partial Q \backslash\{r=0\}$. According to Lemma 4 the partial solutions

$$
J_{m}^{(1)}(r, z) \cos m \varphi, \quad m \in \mathbb{N}_{0}
$$

and

$$
J_{m}^{(2)}(r, z) \sin m \varphi, \quad m \in \mathbb{N},
$$

of equation (2) cannot attain any extremum on the line of degeneracy $r=0$; therefore, the series (45) converges uniformly in $Q_{0}$ and its sum $u_{3}(r, \varphi, z)$ represents the solution of equation (2) from the class $C^{2}\left(Q_{0}\right) \cap C(\bar{Q} \backslash\{r=0\})$, i.e., $u_{3}(r, \varphi, z)$ is the solution of Problem D1. In view of Lemma 4, we have the same estimate (35) for solution $u_{3}$ as for solution $u_{1}$ and $u_{2}$. This implies the uniqueness of the solution $u_{3}$ of Problem D1.

Thus the following theorem holds.

Theorem 3 Let $\alpha=1$. Assume that conditions of Theorem 2 hold. Then Problem D1 has the unique solution $u_{3} \in C^{2}\left(Q_{0}\right) \cap C(\bar{Q} \backslash\{r=0\})$ which can be represented by (45).

So we obtain all exact solutions of Problem D1 subject to the type of degeneracy of equation (2).

\section{Conclusions concerning Problem D1}

It follows from the above that:

1. To the end of well-posedness of Problem D1, the behavior of boundary functions in the vicinity of the points $P_{0}$ and $P_{H}$, in which the line of degeneracy crosses the bases of cylinder $Q$, must be coordinating with the type of degeneracy.

2. The structure of the solutions of Problem D1 and their continuity on the degeneration line depends on the type of degeneracy of equation (2).

3. In the case of the weak degeneracy $(\alpha<1)$, the solution of Problem D1 is continuous on the line of degeneracy if only the boundary function is equal to zero at the points $P_{0}$ and $P_{H}$.

\section{Competing interests}

The author declares that he has no competing interests.

\section{Acknowledgements}

I would like to thank the reviewers for very relevant and significant remarks and comments.

Received: 3 June 2016 Accepted: 5 October 2016 Published online: 19 October 2016

References

1. Rutkauskas, S: The Dirichlet type problem with asymptotic conditions for an elliptic system degenerating at a point, I. Differ. Equ. 38(3), 405-412 (2002)

2. Rutkauskas, S: The Dirichlet type problem with asymptotic conditions for an elliptic system degenerating at a point, II. Differ. Equ. 38(5), 719-725 (2002)

3. Rutkauskas, S: On the solvability conditions of the first boundary value problem for a system of elliptic equations strongly degenerate at a point. Bound. Value Probl. (2011). doi:10.1186/1687-2770-2011-16

4. Beirão da Veiga, H: On nonlinear potential theory and regular boundary points for the $p$-Laplacian in $N$ space variable. Adv. Nonlinear Anal. 3(1), 45-67 (2014)

5. Castorina, D, Sanchon, M: Regularity of stable solutions to semilinear elliptic equations on Riemannian models. Adv. Nonlinear Anal. 4(4), 295-309 (2015) 
6. Motreanu, D, Radulescu, V: Eigenvalue problems for degenerate nonlinear elliptic equations in anisotropic media. Bound. Value Probl. 2005(2), 107-127 (2005). doi:10.1155/BVP.2005.107

7. Yanushauskas, A: On the Dirichlet problem for the degenerating elliptic equations. Differ. Uravn. 7(1), 166-174 (1971) (in Russian)

8. Kvedaras, B: On the solutions of Dirichlet problem to the degenerated elliptic equation. In: Differential Equations and Their Applications, vol. 24, pp. 29-46. Institute of Mathematics and Cybernetics, Vilnius (1979) (in Russian)

9. Rutkauskas, S: Exact solutions of the Dirichlet problem to the degenerate a line elliptic system. Dokl. AMAN 17(4), 84-88 (2015)

10. Watson, GN: A Treatise on the Theory of Bessel Functions. Cambridge University Press, Cambridge (1995)

11. Kratzer, A, Franz, W: Transcendente funktionen. Akad. Verlagsgeselshaft, Leipzig (1960)

12. Tolstov, GP: Fourier Series. Dover, New York (1976)

13. Bateman, H, Erdelyi, A: Higher Transcendental Functions, vol. 2. McGraw-Hill, New York (1953)

14. Bracewell, R: The Fourier Transform and Its Applications. McGraw-Hill, New York (1999)

15. Gilbarg, D, Trudinger, N: Elliptic Partial Differential Equations. Springer, Berlin (1983)

16. Ladyzhenskaya, OA, Ural'tseva, NN: Linear and Quasi-Linear Equations of Elliptic Type. Nauka, Moscow (1973) (in Russian)

17. Bicadze, AV: Boundary Value Problems to Elliptic Equations of Second Order. Nauka, Moscow (1966) (in Russian)

\section{Submit your manuscript to a SpringerOpen ${ }^{\circ}$ journal and benefit from:}

- Convenient online submission

Rigorous peer review

- Immediate publication on acceptance

- Open access: articles freely available online

- High visibility within the field

- Retaining the copyright to your article 